\title{
Modelling the Spatio-Temporal Concentration of Diesel Particulate Matter in an Underground Mine
}

\author{
$\underline{\text { B.J. Mullins }}^{\text {a b }}{ }^{\text {, R.A. O'Leary }}{ }^{\text {c }}$, A.J.C. King ${ }^{\text {b }}$, K. Rumchev ${ }^{\mathrm{a}}$, D. Bertolatti ${ }^{\mathrm{a}}$,

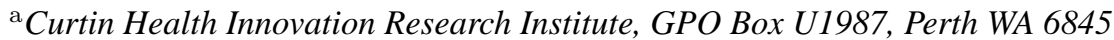 \\ ${ }^{\mathrm{b}}$ Fluid Dynamics Research Group, Curtin University, GPO Box U1987, Perth WA 6845 \\ ${ }^{\mathrm{c}}$ Australian Institute of Marine Science, The UWA Oceans Institute (M096), 35 Stirling Highway, \\ Crawley Western Australia 6009 \\ Email: b.mullins@curtin.edu.au
}

\begin{abstract}
Diesel Particulate Matter (DPM) is an important pollutant, both in industrial areas and cities, and also in underground mines. DPM is essentially the carbonaceous aerosol emitted by diesel engines, with a primary particle size of 10-30 nm, though which rapidly agglomerates to form 100-300 nm aerosols. Most guidelines limit occupational exposure to DPM (measured as elemental carbon) to 100 $\mu \mathrm{g} / \mathrm{m}^{3}$, on an $8-\mathrm{hr}$ averaged basis. However directly assessing worker exposure is both time consuming and expensive. Apart from sampling the exposure of each individual worker, or conducting continuous (and expensive) monitoring, it is difficult to determine if the DPM levels in a workplace will be sufficient to cause DPM exposures above guideline levels. This work has developed a combined particle dynamics and Bayesian regression model, which allows the DPM levels in an underground mine to be predicted both spatially and temporally. The model incorporates known physical effects, (airflow conditions, dispersion, agglomeration), vehicle movement and vehicle emission rates. This enables the model to account for changing (increased) levels of productivity in the mine, a change in the vehicle fleet, or other such factors. The model has been validated against a monitoring study performed in the mine.
\end{abstract}

Keywords: Diesel Particulate Matter (DPM); Diesel Soot; Elemental Carbon; Spatio-Temporal Modelling; Occupational Exposure, Bayesian regression. 


\section{INTRODUCTION}

Exhaust particles emitted from in-use diesel vehicles in underground mining operations are a major source of ultrafine carbonaceous particles, which have the potential to produce adverse health effects. The particulate fraction of diesel exhaust consists of fine particles (typically aerodynamic diameter $<2.5 \mu \mathrm{m}$ ) including a high number of ultrafine particles (diameter $<0.1 \mu$ ) that include reactive organic and transition metal components (Wichmann, 2007).

Particles in the ultrafine size range have attracted significant scientific and medical attention since research shows that for equivalent masses of inhaled particles, ultrafine particles provide a greater surface area for adsorption of potentially toxic substances and metal agents. Further to this, ultrafine particles also posess a higher deposition probability particularly in small airways and the alveolar region of the lungs than coarse particles. With regards to health outcome, the small particle size of diesel particulate matter (DPM) is considered a significant health issue as such particles can penetrate deep in the lungs (Muehlfeld et al., 2008; Die, 1995; Pope et al., 2002). In 1988, the National Institute of Occupational Safety and Health (NIOSH) (Car, 1988) recommended that diesel exhaust be regarded as a 'potential occupational carcinogen'. Similarly, in 1989, the International Agency for Research on cancer declared that 'diesel engine exhaust is probably carcinogenic to human[s]' (Mon, 1989). Measurements have shown that underground miners can be exposed to over 100 times the typical environmental concentrations of diesel particulate matter (DPM) and over 10 times the concentration measured in other work environments where diesel engines are common (Cantrell and Watts, 1997; Cash and Baughman, 2005; Bugarski, Bugarski). Based on the current research evidence, the US National Research Council has concluded that the NIOSH Research Mining Program, related to exposure of DPM and related health effects, is considered as a high priority with a rating of four on a five-point scale (Min, 2007). Furthermore, evidence is increasingly being accumulated which suggests that nanoparticles (such as diesel soot which typically has a primary particle size $<50 \mathrm{~nm}$ ) affect health as a function of surface area rather than mass.

Continual measurement and monitoring of DPM levels - both ambient levels in the workplace (mine) and worker exposure levels, are essential. However, measurement of these levels continuously and/or in realtime are not economical for most workplaces due to the cost of the apparatus. Current standards require occupational exposure to DPM (as elemental carbon, EC) to be assessed gravimetrically using personal samplers. However this method requires laboratory analysis post sample collection, which means that days or weeks will typically elapse between worker exposure and mitigating actions to reduce DPM levels. In addition to this, there is a disconnect between ambient levels and worker exposure, due to the differing nature of the tasks undertaken in the workplace (e.g. some workers may spend most of their shift in an air conditioned vehicle).

It would therefore be advantageous to model DPM levels in a workplace (e.g. an underground mine), in order to allow worker exposure to be predicted either in advance or in realtime, allowing prevention or immediate mitigation of the exposure.

The experimental component of this study assessed personal and environmental exposures of diesel particulate matter (DPM) in an underground mine using various real-time sampling apparatuses. These data were then used to validate models for DPM exposure.

The modelling component of this work developed a spatially resolved model to predict ambient DPM levels in an underground mine based on known vehicle specifications and emission data, measured and predicted airflow and particle attenuation rates. A second model was developed using Bayesian logistic regression to predict worker exposure levels based on ambient DPM levels and job type.

\section{Methods}

\subsection{Experimental}

The study was undertaken in a large underground mine in north-western Australia. The experimental method was designed to: (a) accurately measure DPM in realtime at representative locations, (b) collect size-fractioned DPM and other particulate for gravimetric measurement and analysis, (c) determine worker exposure levels. 
The experimental apparatus consisted of, a NanoMOUDI 125B multi-stage low pressure impactor (MSP, Shoreview, MN, USA), a Scanning Mobility Particle Sizer (SMPS) consisting of a TSI 3081 Differential Mobility Analyzer (DMA) and TSI 3775 Condensation Particle Counter (CPC) (TSI, Shoreview, MN, USA), a TSI DustTrak 8533 DRX, with a TSI P-Trak, TSI Velocicalc-Plus used to obtain air velocity, temperature and humidity measurements.

Four SKC air pumps and filters were utilized for personal monitoring of mine-worker exposures. NIOSH method 5040 (Elemental Carbon) was applied to quantify elemental carbon (EC) via aThermal-optical analyzer.

Samples collected using the NanoMOUDI were analyzed for elemental composition by Scanning Electron Microscopy (SEM) Analysis and performed by the ChemCentre of WA. Analysis of organic species and PAHs was also undertaken.

Samples were taken at a height equivalent to worker head height. Sampling commenced at least 30 minutes after the vehicle containing the sampling equipment was placed into position.

Measurement sites were established at 4 representative locations within the mine. Site 1 (Cell 2 in DPM model) - The main fresh air inlet (baseline measurement) for the mine. Site 2 (Cell 60 in DPM model) - A dead-end passage near the base of the tunnel network with limited airflow. Site 3 (Cell 41 in DPM model) - A central area of the mine where material extraction was actively occurring (also where an electrical substation temporarily reduced airflow) Site 4 (Cell 70 in DPM model) - A site near no. 3 (of 4) air outlet from the mine. Measurements were also taken above ground to compare with the fresh air inlet values.

At each site 1-4, measurements were taken continuously for a period of 6-14 hours. During each measurement, the NanoMOUDI, DustTrak and P-Trak were set to measure continuously. The SMPS was set to perform repeated scans between $15 \mathrm{~nm}$ and $950 \mathrm{~nm}$ at intervals of approximately 15 minutes. At the end of each measurement the equipment was taken to a clean laboratory on the surface where samples/filters could be removed and weighed, and data downloaded, etc.

At the start of each working shift, 3 workers were fitted with personal samplers, selected based on their proximity to the sampling location. The 4th sampler was used to record ambient levels. The samplers were collected after 8 hours and the filters removed for analysis. For the purposes of the (Bayesian regression) model development, samples from a previous measurement campaign were also utilised.

For the time period where samples were being collected, information on vehicle movements and vehicle locations were obtained from the mine telemetry/logistics system. This made it possible to track vehicle movement throughout the mine (including past the sampling locations).

\subsection{Modelling}

A particle dynamics model was developed in order to predict ambient DPM levels based on spatiotemporal vehicle operations within the mine. In order to simplify the model, a discrete network of tunnel segments or 'Cells' was used to construct the model. Approximately $7 \mathrm{~km}$ of tunnel was split into 70 discrete cells of $100 \mathrm{~m}$ in length. For each of these cells, approximate cross sectional areas and calculated or estimated flow rates were recorded, in order to determine air exchange rates.

Based on vehicle movement information from the mine telemetry system, the duration of each type of (diesel) mine vehicle (truck, loader, $4 \times 4$ ) was determined for each Cell. From these and known vehicle specifications, DPM loading per cell could be calculated, which was then converted to average concentrations in each cell based on calculated air exchange rates. Transport to downstream Cells was included using an exponential decay function to account for collection of DPM on tunnel walls/floor. In order to determine the relationship between ambient DPM levels and individual worker (DPM) exposure levels, a Bayesian Linear Regression Model was implemented (Gelman et al., 1995). The model covariates were: measured ambient DPM levels; job type (see below); whether the worker(i.e. job type) operates a diesel or electric vehicle and whether the worker normally works inside or outside the vehicle. All covariates were assumed to have a non-informative normal prior. Analysis was conducted using Winbugs. 


\section{Results}

Table 1 shows the types of diesel vehicle types in use in the underground mine, namely, Loaders, haulage Trucks and $4 \times 4$ utilities. Specifications for the engines used in the vehicles and emissions standards were obtained from the manufacturers (www.miningandconstruction.sandvik.com/ and www.toyota.com.au ). Other vehicles such as drill rigs operate within the mine also, however these are electrically powered so were excluded from the dataset.

Table 1. Types of diesel vehicle types in use in the underground mine

\begin{tabular}{|c|c|c|c|}
\hline Vehicle Type & Power $(\mathrm{kW})$ & $33 \%$ Duty Power & Emission Std. \\
\hline Truck & 375 & 123.75 & Euro 3 \\
Loader & 220 & 72.6 & Euro 3 \\
$4 x 4 / G P$ & $\approx 150$ & 49.5 & Euro 3 \\
\hline
\end{tabular}

One third of peak engine power rating was selected as a representative average operational duty for modelling purposes. The $4 \times 4$ utilities in use vary in engine power, so this value was averaged across vehicle types/models in use. All vehicles conform to Euro 3 emissions standards - maximum $0.13 \mathrm{~g} / \mathrm{kWh}$ DPM, and the 85th percentile of this value was selected as representative.



Figure 1. Measured and modelled ambient DPM levels per mine cell

The mine tunnels (representing a significant sub-section of the mine) were segregated into 70 Cells of approximately $100 \mathrm{~m}$ in length each. Air exchange rates were calculated based on measured (and interpolated values based on measurements and tunnel intersections/diameter changes. Values varied between 
6 and 72 air changes per hour (high readings were at the fresh air inlet which is not typically traversed by people or vehicles.

Mine telemetry data taken during the experimental campaign was used to determine the approximate location of each vehicle operating in the mine, and thereby apportion it to a Cell. Each vehicle was essentially treated as a point source within each cell, however cells were not resolved below $100 \mathrm{~m}$ resolution.

Figure 1 shows the modeled $8 \mathrm{hr}$ average DPM levels per mine cell and corresponding 6-10 hr average measurement values for 4 sites. It can be seen that the agreement between measured and modeled points (circled) is quite good. However, a larger set of measured locations would be advantageous. It would also be useful to ascertain if the peak values found during modelling are representative, or if rapid agglomeration and collection occurs in these cases - e.g. loaders travel in reverse as frequently as forwards, so could be expected to collect significant quantities of DPM on the outside of the vehicle. The horizontal line shows the workplace ( $8 \mathrm{hr}$ average) exposure guideline - which is evidently exceeded in parts of the mine.

Measured ambient DPM values (converted to EC) were tabulated, together with corresponding DPM exposures (measured as EC) of workers operating in the immediate vicinity $(<30 \mathrm{~m}$ from stationary sampler). Also included in the dataset was if the worker operates a diesel or electric vehicle, and if the worker spends the majority of their work inside an (air conditioned) vehicle cab or in the ambient (underground) air (Note that personal observations have found that some workers prefer to drive their vehicles with the windows open - which would increase their exposure levels).

Worker categories were: $\mathrm{L}(1)=$ Loader Driver (Diesel, normally In vehicle) $\mathrm{T}(2)=$ Haul Truck Driver (Diesel, normally In vehicle) $\mathrm{D}(3)=$ Driller/Assistant Driller (Electric, normally In vehicle) $\mathrm{E}(4)=$ Explosives Technician (Diesel, normally Outside vehicle) $\mathrm{M}(5)=$ Underground Maintenance Technician/Mechanic (Diesel, normally Outside vehicle) C $(6)=$ Concreter (Diesel, normally Outside vehicle)

A Bayesian logistic regression was conducted to determine if a significant correlation existed between ambient and worker exposure, when job factors are considered. The following relationship was obtained:

$$
\begin{array}{r}
\ln (D P M W)=-4.289+16.32 \times D P M A-13.12 \times V-5.023 \times W+0 \times D+13.85 \times L \\
+13.13 \times T+17.92 \times E+18.13 \times M+18.0 \times C
\end{array}
$$

Where, DPMW $=$ worker DPM exposure DPMA $=$ ambient DPM level $\mathrm{V}=$ Vehicle type $($ Diesel $=1$, Electric $=0) \mathrm{W}=$ Normal work location $($ Outside vehicle $=1$, Inside vehicle $=0$ ) The remaining parameters are the job type (each worker class not filled will be assigned a value of 0 which will render the term equal to 0 ).

Figure 2 shows the observed vs predicted results for Equation 2. The agreement is extremely good, despite the small dataset. A larger sample for full validation would be optimal in the future.

\subsection{Conclusions and Recommendations}

This work presents both an extensive investigation into DPM levels in underground mines, as well as presenting a promising approach to simulate and manage DPM levels in realtime on an operational level. Both models could benefit from increased temporal and spatial resolution and large (experimental) datasets for validation purposes.

Very high levels of DPM were found in the mine, which are likely to only increase once production levels are increased. The mine was operating at $1 / 3$ capacity during the measurement campaign.

It is hoped that this model will be beneficial in preventing future DPM exceedences among mine workers.

\section{ACKNOWLEDGMENTS}

The authors would like to acknowledge the mine staff for assistance with the sampling. 




Figure 2. Observed Worker DPM Exposure vs Bayesian Regression Predicted Exposure. Values in $\mathrm{mg} / \mathrm{m}^{3}$

\section{REFERENCES}

(1988). Carcinogenic effects of exposure to diesel exhaust; national institute of occupational safety and health (niosh), current intelligence bulletin 50 .

(1989). Monographs on the evaluation of carcinogenic risks to humans diesel and gasoline engine exhausts and some nitroarenes.

(1995). Diesel exhaust: A critical analysis of emissions, exposures, and health effects. a special report of the institute's working group; health effects institute web site.

(2007). Mining Safety and Health Research at NIOSH: Reviews of Research Programs of the National Institute for Occupational Safety and Health. Washington DC: NISBN: 0-309-66636-8, National Academies Press.

Bugarski, S. . P. Implementation of diesel particulate filter technology in underground metal and nonmetal mines.

Cantrell, B. and W. Watts (1997). Diesel exhaust aerosol: Review of occupational exposure. Applied Occupational and Environmental Hygiene 12, 1019-1026.

Cash, D. and W. Baughman (2005). DPM exposure in metal and nonmetal mines in the United States 2002-2005 and the 2005 Final Rule on the Interim Limit. Proceeding of Mining Diesel Emissions Conference (MDEC), Markham, Ontario, October 12-14, 2005.Quoted in Bugarski, Schnakenberg \& Patts, p. 127. 
Gelman, A., J. B. Carlin, H. S. Stern, and D. B. Rubin (1995). Bayesian Data Analysis (1st ed.). Florida: Chapman \& Hall/CRC.

Muehlfeld, C., B. Rothen-Rutishauser, F. Blank, D. Vanhecke, M. Ochs, and P. Gehr (2008). Interactions of nanoparticles with pulmonary structures and cellular responses. American Journal of PhysiologyLung Cell Molecular Physiology 294, 817-829.

Pope, A., R. Burnett, M. Thun, E. Calle, D. Krewski, K. Ito, and G. Thurston (2002). Lung cancer, cardiopulmonary mortality, and long-term exposure to fine particulate air pollution. Journal of the American Medical Association 287, 1132-1141.

Wichmann, H. (2007). Diesel exhaust particles. Inhalation Toxicology 19(Suppl.1), 241-244. 